\section{An Examination of Stewardship Interventions by Major Category in an Urban Academic Medical Center}

To the Editor-Antimicrobial stewardship programs (ASPs) have been developed to promote proper use of antimicrobials. The primary goals of ASPs are to enhance patient safety and improve quality of care by limiting inappropriate use and optimizing dosing, route, and duration of antimicrobial therapy. ${ }^{1}$ Antimicrobials account for upward of $30 \%$ of hospital pharmacy budgets, and ASPs can save $\$ 200,000-\$ 900,000$ annually. ${ }^{1,2}$ Additionally, randomized clinical trials have shown that computerized surveillance systems result in significant savings in cost and time. ${ }^{3}$ One-quarter of nosocomial infections occur in patients in intensive care units, and nearly $70 \%$ of infections that occur in the intensive care unit are due to organisms that are resistant to antibiotics. ${ }^{4}$ ASPs can employ interventions designed to promote appropriate antimicrobial coverage and reduce antimicrobial selective pressure.

Virginia Commonwealth University is an 820 -bed academic medical center at which the ASP is comprised of a 0.5 full-time equivalent (FTE) physician epidemiologist and 2 pharmacists who together share 1.0 FTE. The program uses a computer surveillance program, Safety Surveillor, which screens electronic order entry and microbiology data and generates alerts when predetermined parameters are violated. The parameters designed to promote appropriate antimicrobial coverage include bloodstream, urine, and lower respiratory tract microbe susceptibility and/or drug mismatch; when organisms are isolated without antimicrobial coverage; or when a suboptimal antimicrobial is selected. The parameters de- signed to decrease antimicrobial selective pressure include situations when a narrower spectrum antimicrobial is indicated; after 7 days of broad-spectrum gram-negative coverage; after 96 hours of vancomycin without isolation of methicillinresistant Staphylococcus aureus and double coverage of anaerobic, fungal, gram-positive, or gram-negative organisms. When any of the predetermined parameters are violated, an alert is generated and reviewed by an ASP pharmacist. If, on the basis of chart review, the pharmacist decides that an intervention is warranted, she then intervenes by contacting the treating clinician. For each intervention, ASP concurrence is documented if the treating physician accepts the ASP's original recommendation or if the ASP pharmacist agrees with either the original or an alternative management plan. Interventions are classified into those designed to promote appropriate antimicrobial coverage and those designed to decrease antimicrobial selective pressure (Table 1). Data generated from the ASP interventions from February 2007 through December 2009 were analyzed to assess ASP concurrence with ultimate management for adult inpatient services.

There were a total of 2,126 interventions over the 3-year period. Of these interventions, $1,679(79 \%)$ were to decrease antimicrobial selective pressure, compared with 447 (21\%) to promote appropriate antimicrobial coverage. Overall, there was $72 \%$ concurrence $(1,214$ of 1,679 interventions) with interventions that were designed to reduce antimicrobial selective pressure versus $85 \%$ concurrence ( 381 of 447 interventions) with interventions that were designed to promote appropriate antimicrobial coverage $(P<.01$; Table 1$)$.

We reviewed ASP data collected over a 3-year period and compared the frequency and concurrence between intervention types. Over the 35 months for which data were collected,

ASP agreed with management, no.

$(\%)$ of interventions

\begin{tabular}{|c|c|c|c|}
\hline \multirow[b]{2}{*}{ Intervention, by type } & & & \\
\hline & Yes & No & Total \\
\hline \multicolumn{4}{|l|}{ Interventions to promote appropriate antimicrobial coverage } \\
\hline Organisms present without antibiotic coverage & $232(52)$ & $58(13)$ & $290(65)$ \\
\hline Organisms present on culture that are resistant to current antimicrobiala & $85(19)$ & $2(0.5)$ & $87(19.5)$ \\
\hline Suboptimal antimicrobial selection & $64(14)$ & $6(1.5)$ & $70(15.5)$ \\
\hline Overall & $381(85)$ & $66(15)$ & $447(100)$ \\
\hline \multicolumn{4}{|l|}{ Interventions to reduce antimicrobial selective pressure } \\
\hline Prolonged duration of therapy & $390(23)$ & $166(10)$ & $556(33)$ \\
\hline No indication for current antimicrobial & $392(23)$ & $139(8)$ & $531(31)$ \\
\hline Double coverage & $334(20)$ & $129(8)$ & $463(28)$ \\
\hline Narrower spectrum of action indicated & $98(6)$ & $31(2)$ & $129(8)$ \\
\hline Overall & $1,214(72)$ & $465(28)$ & $1,679(100)$ \\
\hline
\end{tabular}

NOTE. ASP, antimicrobial stewardship program.

a Includes bloodstream, urine, lower respiratory tract cultures. 


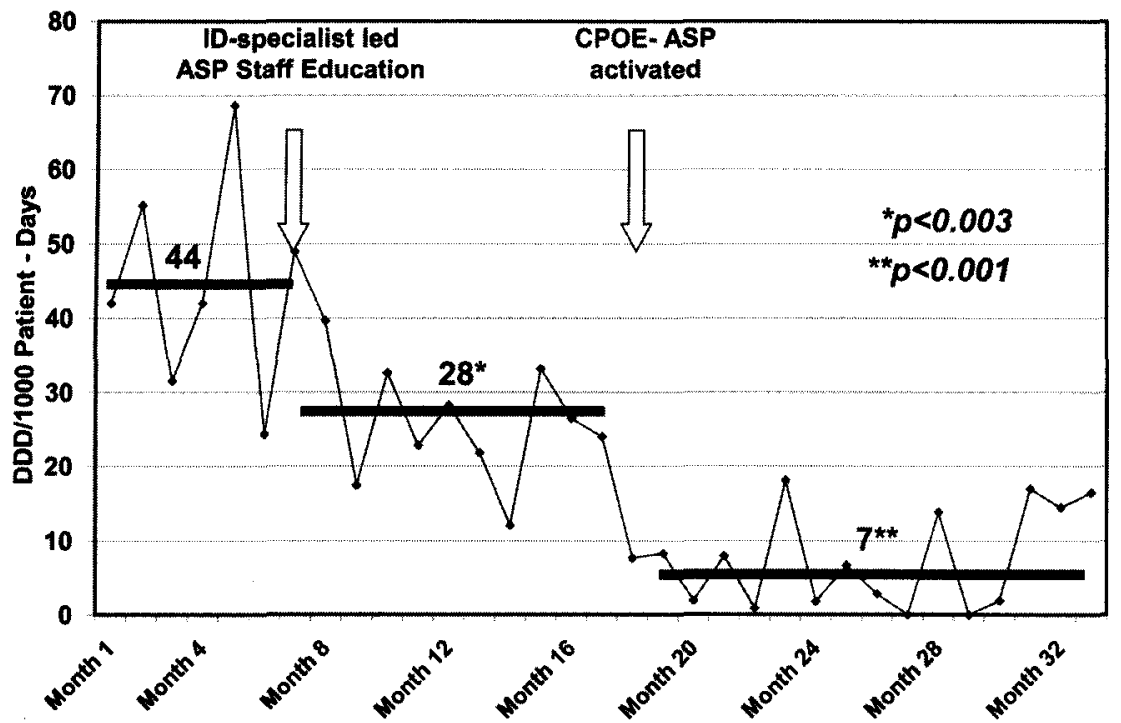

FIGURE 1. Linezolid use over 36 months, covering the periods before and during antimicrobial stewardship (ASP) education and after computer physician/provider order entry (CPOE) activation.

TABLE 1. Antibacterial Courses Used in Hospital da Luz during January 2011

\begin{tabular}{lccr}
\hline & Therapeutic $^{\mathrm{a}}$ & Prophylactic $^{\mathrm{a}}$ & Total $^{\mathrm{b}}$ \\
\hline Tetracyclines & $8(100)$ & $\ldots$ & $8(0.8)$ \\
Penicillins & $98(93.3)$ & $7(6.7)$ & $105(11.0)$ \\
First-generation cephalosporins & $6(1.2)$ & $511(98.8)$ & $517(54.3)$ \\
Second-generation cephalosporins & $13(21.3)$ & $48(78.7)$ & $61(6.4)$ \\
Third-generation cephalosporins & $54(93.1)$ & $4(6.9)$ & $58(6.1)$ \\
Carbapenems & $18(100)$ & $\ldots$ & $18(1.9)$ \\
Sulfonamides and trimethoprim & $6(100)$ & $\ldots$ & $6(0.6)$ \\
Macrolides & $44(97.8)$ & $1(2.2)$ & $45(4.7)$ \\
Lincosamides & $3(75.0)$ & $1(25.0)$ & $4(0.4)$ \\
Aminoglycoside antibacterials & $12(92.3)$ & $1(7.7)$ & $13(1.4)$ \\
Quinolones & $44(77.2)$ & $13(22.8)$ & $57(6.0)$ \\
Glycopeptide antibacterials & $9(100)$ & $\ldots$ & $9(0.9)$ \\
Imidazole derivatives & $21(42.9)$ & $28(57.1)$ & $49(5.1)$ \\
Other antibacterials & $2(100)$ & $\ldots$ & $2(0.2)$ \\
$\quad$ Total & 338 & 614 & 952 \\
\hline
\end{tabular}

Note. Date are no. (\%).

a Percentage out of the total therapeutic class.

b Percentage out of the total antibacterial use.

the number of interventions to decrease antimicrobial selective pressure was 4 times greater than the number of interventions to promote proper antimicrobial coverage. ASPprovider concurrence with recommendations aimed at appropriate coverage was $85 \%$ and was only $72 \%$ for antimicrobial guidance that limits selective pressure. For all interventions, regardless of type, there was $75 \%$ concurrence.

Evidence suggests that ASPs can decrease the use of parenteral broad-spectrum antibiotics, even in the setting of increasing patient acuity, in addition to improving patient safety by decreasing the rates of nosocomial infection due to Clostridium difficile and other drug-resistant organisms. ${ }^{5}$ By in- tervening and providing expert consultation, ASPs can guide clinicians in practices that reduce antimicrobial selective pressure and improve the appropriate use of antimicrobial drugs.

\section{ACKNOWLEDGMENTS}

Potential conflicts of interest. All authors report no conflicts of interest relevant to this article. All authors submitted the ICMIE Form for Disclosure of Potential Conflicts of Interest, and the conflicts that the editors consider relevant to this article are disclosed here. 
James Pellerin, MD; ${ }^{1}$ Michael Edmond, MD, MPH, MPA; ${ }^{1}$ Gonzalo Bearman, MD, MPH; ${ }^{1}$ Kimberly Lee, PharmD; ${ }^{1}$ Michael P. Stevens, MD, MPH

Affiliations: 1. Virginia Commonwealth University Medical Center, Richmond, Virginia.

Address correspondence to James Pellerin, MD, Virginia Commonwealth University Medical Center, 1300 East Marshall Street, P.O. Box 980019, Richmond, Virginia 23298 (jpelleri@mcvh-vcu.edu).

Presented in part: 2011 Annual Meeting of the Society for Healthcare Epidemiology of America; Dallas, Texas; April 3, 2011 (Abstract 4362). Infect Control Hosp Epidemiol 2012;33(4):432-434

(C) 2012 by The Society for Healthcare Epidemiology of America. All rights reserved. 0899-823X/2012/3304-0023\$15.00. DOI: 10.1086/664921

\section{REFERENCES}

1. Dellit T, Owens R, McGowan J, et al. Infectious Diseases Society of America and the Society for Healthcare Epidemiology of America guidelines for developing an institutional program to enhance antimicrobial stewardship. Clin Infect Dis 2007;44:159-177.

2. John JF Jr, Fishman NO. Programmatic role of the infectious disease physician in controlling antimicrobial costs in the hospital. Clin Infect Dis 1997;24:471-485.

3. McGregor J, Weekes E, Forrest G, et al. Impact of a computerized clinical decision support system on reducing inappropriate antimicrobial use: a randomized controlled trial. J Am Med Inform Assoc 2006;13:378-384.

4. Burke J. Infection control: a problem for patient safety. New Engl J Med 2003;348:651-656.

5. Carling P, Fung T, Killion A, Terrin N, Barza M. Favorable impact of a multidisciplinary antibiotic management program conducted during 7 years. Infect Control Hosp Epidemiol 2003;24:699-706.

\section{The Impact of an Infectious Diseases Specialist-Directed Computerized Physician Order Entry Antimicrobial Stewardship Program Targeting Linezolid Use}

To the Editor-The utilization of various antimicrobial stewardship program (ASP) strategies such as prior authorization, prescriber feedback and education, and antibiotic order forms have demonstrated favorable impacts on antibiotic utilization in academic settings. ${ }^{1-4}$ To facilitate the implementation of ASPs, institutions have designed computer systems allowing physician/provider order entry (CPOE). CPOE allows direct entry of medical orders by authorized healthcare providers; this has the benefit of reducing errors by minimizing the ambiguity of handwritten orders, with greater benefits seen with the combination of CPOE and clinical decision support tools. ${ }^{5}$ In order to assess the potential impact of physician intervention on our community hospital-based, pharmacydirected ASP, we undertook a prospective evaluation of li- nezolid use following the addition of an infectious diseases (ID) physician to the program. The subsequent addition of a customized CPOE-ASP order entry template incorporating a linezolid decision algorithm provided an opportunity to monitor its potential additional impact over the subsequent 16 months.

In our 214-bed suburban nonacademic hospital, linezolid use was measured during a 32-month period from January 2008 to September 2010. The utilization formula combined a standardized defined daily dose (DDD) of $1,200 \mathrm{mg}$ as recommended by the World Health Organization with hospital pharmacy purchasing data and hospital patient-days (PTD) to calculate a monthly DDD per 1,000 PTD.

Prior to implementation of the CPOE-ASP, a primary intervention consisting of ID physician educational activities represented the only new intervention that had the potential for impacting linezolid use. Shortly prior to implementation of the CPOE system, linezolid usage guidelines based on Food and Drug Administration-approved indications ${ }^{6}$ along with additional evidence-based recommendations approved by a local committee of clinical pharmacists and ID specialists were developed specifically for our CPOE system. Recommendations regarding alternative antibiotics with their dosages and rationale for use as well as hyperlinked references were included in the order entry form. All providers ordering antibiotics were identified and educated on the CPOE system and the antibiotic guidelines. In addition, a linezolid utilization audit was performed over two 5-month periods during the preintervention and CPOE-ASP periods by clinical pharmacists to determine whether linezolid orders reflected institution-approved indications.

The pharmacy provided information on the direct cost of linezolid during the periods studied. Baseline linezolid use over the 7 months prior to ID physician leadership involvement in the hospital's ASP averaged $44 \mathrm{DDD} / 1,000$ PTD (Figure 1). Following ID physician involvement in the program and education of the medical staff, over a 9-month period linezolid use fell to $28 \mathrm{DDD} / 1,000$ PTD $(P<.003$, Student $t$ test). A further decrease to a mean of $7 \mathrm{DDD} / 1,000$ PTD was realized and sustained over a subsequent 16-month period following CPOE implementation in the setting of ongoing physician involvement $(P<.001$ from baseline, Student $t$ test). Examination of the proportion of nonappropriate linezolid use by the pharmacist-based audit confirmed a significant decrease in linezolid orders that deviated from institutional guidelines from $77 \%$ ( 26 of 34 orders) to $11 \%$ (1 of 13 orders; $P<.003$, Fisher exact test).

A review of the number of vancomycin-resistant enterococci (VRE) infections (based on VRE-positive cultures and the number of VRE-positive isolation rooms requested) demonstrated no changes during the study implementation. The length of stay for patients with skin/soft tissue infections was unchanged before and after CPOE implementation (data not shown). During the study there were no clear trends in overall 